\title{
Increasing the chromatic number of a random graph
}

\author{
Noga Alon* And Benny Sudakov ${ }^{\dagger}$
}

\begin{abstract}
What is the minimum number of edges that have to be added to the random graph $G=G_{n, 0.5}$ in order to increase its chromatic number $\chi=\chi(G)$ by one percent? One possibility is to add all missing edges on a set of $1.01 \chi$ vertices, thus creating a clique of chromatic number $1.01 \chi$. This requires, with high probability, the addition of $\Omega\left(n^{2} / \log ^{2} n\right)$ edges. We show that this is tight up to a constant factor, consider the question for more general random graphs $G_{n, p}$ with $p=p(n)$, and study a local version of the question as well.

The question is motivated by the study of the resilience of graph properties, initiated by the second author and $\mathrm{Vu}$, and improves one of their results.
\end{abstract}

\section{Introduction}

Consider the probability space whose points are graphs on $n$ labeled vertices, where each pair of vertices forms an edge, randomly and independently with probability $p$. The random graph $G_{n, p}$ denotes a random point in this probability space. This concept is one of the central notions in modern discrete mathematics and it has been studied intensively during the last 50 years. By now, there are thousands of papers and two excellent monographs by Bollobás [5] and by Janson et al. [9] devoted to random graphs and their diverse applications. The subject of the theory of random graphs is the investigation of the asymptotic behavior of various graph parameters. We say that a graph property $\mathcal{P}$ holds asymptotically almost surely (a.a.s.) if the probability that $G_{n, p}$ has $\mathcal{P}$ tends to one as $n$ tends to infinity.

One of the most important parameters of the random graph $G_{n, p}$ is its chromatic number, which we denote by $\chi\left(G_{n, p}\right)$. Trivially for every graph $\chi(G) \geq|V(G)| / \alpha(G)$, where $\alpha(G)$ denotes the size of the largest independent

\footnotetext{
${ }^{*}$ Research supported in part by an ERC Advanced grant, by a USA-Israeli BSF grant, and by the Hermann Minkowski Minerva Center for Geometry in Tel Aviv University.

${ }^{\dagger}$ Research supported in part by NSF CAREER award DMS-0812005 and by a USA-Israeli BSF grant.
} 
set in $G$. It can be easily shown, using first moment computations, that a.a.s. $\alpha\left(G_{n, p}\right) \leq 2 \log _{b}(n p)$, where $b=1 /(1-p)$ (all logarithms in this paper are in the natural base $e$ ). This provides a lower bound on the chromatic number of the random graph, showing that $\chi\left(G_{n, p}\right) \geq \frac{n}{2 \log _{b}(n p)}$. The problem of determining the asymptotic behavior of $\chi\left(G_{n, p}\right)$, posed by Erdős and Rényi in the early $60 \mathrm{~s}$, stayed for many years as one of the major open problems in the theory of random graphs, until its solution by Bollobás [4], using a novel approach based on martingales that enabled him to prove that a.a.s. $\chi\left(G_{n, p}\right)=(1+o(1)) \frac{n}{2 \log _{b}(n p)}$ for dense random graphs. Later Euczak [14] showed that this estimate also holds for all values of $p \geq c / n$. In this paper we strengthen these classical results, by showing that the chromatic number remains $(1+o(1)) \frac{n}{2 \log _{b}(n p)}$ even if we are allowed to add to $G_{n, p}$ any set of not too many additional edges. To describe the main results it is convenient to use the framework of resilience, introduced by Sudakov and Vu [15].

A graph property is called monotone increasing (decreasing) if it is preserved under edge addition (deletion). Following [15], we define:

Definition 1.1. Let $\mathcal{P}$ be a monotone increasing (decreasing) graph property.

- The global resilience of $G$ with respect to $\mathcal{P}$ is the minimum number $r$ such that by deleting (adding) $r$ edges from $G$ one can obtain a graph not having $\mathcal{P}$.

- The local resilience of a graph $G$ with respect to $\mathcal{P}$ is the minimum number $r$ such that by deleting (adding) at most $r$ edges at each vertex of $G$ one can obtain a graph not having $\mathcal{P}$.

Intuitively, the question of determining the resilience of a graph $G$ with respect to a graph property $\mathcal{P}$ is like asking, "How strongly does $G$ possess $\mathcal{P}$ ?". Using this terminology, one can restate many important results in extremal graph theory in this language. For example, the classical theorem of Dirac asserts that the complete graph $K_{n}$ has local resilience $\lfloor n / 2\rfloor$ with respect to having a Hamilton cycle. In [15], the authors have initiated the systematic study of global and local resilience of random and pseudo-random graphs. They obtained resilience results with respect to various properties such as perfect matching, Hamiltonicity, chromatic number and having a nontrivial automorphism (the last result appeared in an earlier paper with Kim [10]). For example, they proved that if $p>\log ^{4} n / n$ then a.a.s. any subgraph of $G(n, p)$ with minimum degree $(1 / 2+o(1)) n p$ is Hamiltonian. Note that this result can be viewed as a generalization of Dirac's theorem mentioned above, since a complete graph is also a random graph $G(n, p)$ 
with $p=1$. This connection is natural and most of the resilience results for random and pseudo-random graphs can be viewed as generalizations of classical results from graph theory. For additional recent resilience type results, see, e.g., [7, 8, 12, 6, 3].

In [15], Sudakov and $\mathrm{Vu}$ proved that the local resilience of dense $G_{n, p}$ with respect to having chromatic number $(1+o(1)) \frac{n}{2 \log _{b}(n p)}$ is at least $n p^{2} / \log ^{5} n$. The main aim of the present short paper is to obtain the following new bounds on both the global and the local resilience of the chromatic number of the random graph, which substantially improve this result from [15].

Theorem 1.2. Let $\epsilon>0$ be a fixed constant and let $n^{-1 / 3+\delta} \leq p \leq 1 / 2$ for some $\delta>0$. Then a.a.s. for every collection $E$ of $2^{-12} \epsilon^{2} \frac{n^{2}}{\log _{b}^{2}(n p)}$ edges the chromatic number of $G_{n, p} \cup E$ is still at most $(1+\epsilon) \frac{n}{2 \log _{b}(n p)}$.

This shows that the global resilience of $G_{n, p}$ with respect to having chromatic number at most $(1+\epsilon) \frac{n}{2 \log _{b}(n p)}$ is of order $n^{2} / \log _{b}^{2}(n p)$. The result is tight up to a constant factor. Indeed, take an arbitrary set of, say, $n / \log _{b}(n p)$ vertices of the random graph and add to it all the missing edges to make it a clique. This adds less than $\frac{1}{2} n^{2} / \log _{b}^{2}(n p)$ edges but increases the chromatic number by a factor of 2 .

Theorem 1.3. Let $\epsilon>0$ be a fixed constant and let $n^{-1 / 3+\delta} \leq p \leq 1 / 2$ for some $\delta>0$. Then a.a.s. for every graph $H$ on $n$ vertices with maximum degree $\Delta(H) \leq 2^{-8} \epsilon \frac{n}{\log _{b}(n p) \log \log n}$ the chromatic number of $G_{n, p} \cup H$ is still at most $(1+\epsilon) \frac{n}{2 \log _{b}(n p)}$.

As before, by transforming a subset of $n / \log _{b}(n p)$ vertices of the random graph to a clique, it follows that this result is tight up to the $\log \log n$ factor. Both these theorems show that adding quite large and dense graphs to $G_{n, p}$ has very little impact on its chromatic number. It may be instructive to compare the above two theorems to the following folklore result (see, e.g., [13] Chapter 9).

Fact 1.4. Let $G$ and $H$ be two graphs on the same set of points. Then

$$
\chi(G \cup H) \leq \chi(G) \chi(H),
$$

and there are pairs of $G$ and $H$ such that the equality holds.

The rest of this short paper is organized as follows. In the next section we prove our key technical lemma, which shows that in the random graph 
$G_{n, p}$ the independent sets of nearly maximal size are rather uniformly distributed. Using this lemma we establish Theorems 1.2 and 1.3 in Section 3. The last section of the paper contains some concluding remarks and open questions. Throughout the paper, we systematically omit floor and ceiling signs whenever they are not crucial for the sake of clarity of presentation. We also do not make any serious attempt to optimize the absolute constants in our statements and proofs.

\section{The distribution of independent sets in random graphs}

In this section we prove the statement which will be our main technical tool. It shows that no matter which set of $m$ edges we add to the random graph $G_{n, p}$, a.a.s. there will be an independent set of nearly maximal size which contains only a few of these edges. In order to state our result precisely we need some preliminaries.

Let $n^{-1 / 3+\delta} \leq p \leq 1 / 2$ for some $\delta>0$ and let $k_{0}=k_{0}(n, p)$ be defined by

$$
k_{0}=\max \left\{k:\left(\begin{array}{l}
n \\
k
\end{array}\right)(1-p)^{\left(\begin{array}{c}
k \\
2
\end{array}\right)} \geq n^{4}\right\} .
$$

One can show easily that $k_{0}$ satisfies $k_{0} \sim 2 \log _{b}(n p)$ with $b=1 /(1-p)$. Also, it follows from known results on the asymptotic value of the independence number of $G(n, p)$ (see, e.g., $[9,2])$ that a.a.s. the difference between $k_{0}$ and the independence number of $G(n, p)$ is bounded by an absolute constant, as long as $p(n)$ is in the above range.

Let $\mu$ be the expected number of independent sets of size $k_{0}$ in $G_{n, p}$. Clearly

$$
\mu=\left(\begin{array}{c}
n \\
k_{0}
\end{array}\right)(1-p)^{\left(\begin{array}{c}
k_{0} \\
2
\end{array}\right)} \geq n^{4},
$$

by the definition of $k_{0}$. For a pair $u, v \in G_{n, p}$, let $Z_{u, v}$ be the random variable counting the number of independent sets of size $k_{0}$ in $G_{n, p}$ that contain both $u$ and $v$. Let $\mu_{0}=E\left[Z_{u, v}\right]$, then

$$
\mu_{0}=\left(\begin{array}{c}
n-2 \\
k_{0}-2
\end{array}\right)(1-p)^{\left(\begin{array}{c}
k_{0} \\
2
\end{array}\right)} .
$$

It is easy to see that $\mu_{0} / \mu=(1+o(1)) k_{0}^{2} / n^{2}$.

Let $X$ be the random variable which is equal to the size of a largest collection of independent sets of size $k_{0}$ in the random graph $G_{n, p}$ such that 
no pair of vertices $u, v$ belongs to more than $4 \mu_{0}$ of these sets. We need the following lemma, which shows that with high probability the value of $X$ is concentrated around $\mu$.

\section{Lemma 2.1.}

$$
\operatorname{Pr}[X \leq 3 \mu / 5] \leq e^{-\frac{\mu^{2}}{300 \mu_{0}^{2} n^{2} p}} .
$$

To prove this lemma we will need first to estimate from below the expectation of $X$. For a pair of vertices $u, v$ in $G_{n, p}$ set

$$
Z_{u, v}^{+}= \begin{cases}Z_{u, v}, & Z_{u, v}>4 \mu_{0} \geq 2 \mu_{0} /(1-p) \\ 0, & \text { otherwise. }\end{cases}
$$

We also define $Z^{+}=\sum_{u, v} Z_{u, v}^{+}$. This random variable has been considered before in [11], where the authors studied the probability that the random graph $G_{n, p}$ contains an independent set of size $k_{0}$. We will need the following claim, proved in that paper.

Proposition 2.2. $\mathbb{E}\left[Z^{+}\right]=o(\mu)$.

From this proposition we can immediately deduce the following bound on the expectation of $X$.

Corollary 2.3. Let $X$ be the size of a largest collection of independent sets of size $k_{0}$ in the random graph $G_{n, p}$ such that no pair of vertices belongs to more than $4 \mu_{0}$ of these sets. Then $\mathbb{E}[X]=(1-o(1)) \mu$.

Proof. Let $\mathcal{F}$ be the collection of all independent sets in $G_{n, p}$ of size $k_{0}$. By the definition of $k_{0}$, we have that $\mathbb{E}[|\mathcal{F}|]=\mu$. For every pair of vertices $u, v$ which is contained in more than $4 \mu_{0}$ independent sets of size $k_{0}$, delete all these sets from $\mathcal{F}$. Note that for every pair of vertices $u, v$ we deleted at most $Z_{u, v}^{+}$sets and therefore the total number of deleted sets is at most $Z^{+}$. It is easy to see that the remaining independent sets cover every pair of vertices at most $4 \mu_{0}$ times and therefore their number is at most $X$. By Proposition 2.2, this implies that

$$
\mathbb{E}[X] \geq \mathbb{E}[|\mathcal{F}|]-\mathbb{E}\left[Z^{+}\right]=(1-o(1)) \mu .
$$

This completes the proof, since clearly $\mathbb{E}[X] \leq \mu$.

Let $\mathcal{I}$ be a largest collection of independent sets of size $k_{0}$ in the random graph $G_{n, p}$ such that no pair of vertices belongs to more than $4 \mu_{0}$ of these sets and recall that $X=|\mathcal{I}|$. Note that when we connect by a new edge a pair of non-adjacent vertices $u, v$ of $G_{n, p}$ we can decrease the value of $X$ only 
by the number of independent sets in $\mathcal{I}$ which contain $u, v$. By definition, this is at most $4 \mu_{0}$. Now suppose we delete an existing edge $(u, v)$ of the random graph. Although this might create many new independent sets of size $k_{0}$, they all contain $u, v$ and we can include only at most $4 \mu_{0}$ of them in $\mathcal{I}$. Hence also in this case the value of $X$ changes by at most $4 \mu_{0}$, i.e., $X$ is a so called $4 \mu_{0}$-Lipschitz function. Now to finish the proof of Lemma 2.1 we apply a concentration inequality for such functions, proved by Alon, Kim and Spencer ([1], see also [2], Theorem 7.4.3). They considered a random variable $Y$ given on the space generated by mutually independent $0 / 1$ choices such that probability that a choice $i$ is one is $p_{i}$. Let $c_{i}$ be such that changing choice $i$ can change $Y$ by at most $c_{i}, C=\max _{i} c_{i}$ and let the total variance satisfy $\sum_{i} p_{i}\left(1-p_{i}\right) c_{i}^{2} \leq \sigma^{2}$. Then if $a C<2 \sigma^{2}$ for some positive $a$, then

$$
\operatorname{Pr}[Y-\mathbb{E}[Y]<-a] \leq e^{-a^{2} /\left(4 \sigma^{2}\right)} .
$$

Proof of Lemma 2.1. As we already mentioned $X$ is a $4 \mu_{0}$-Lipschitz random variable, which depends on $\left(\begin{array}{l}n \\ 2\end{array}\right)$ random choices for the edges of $G_{n, p}$. This implies that the total variance is at most $16 \mu_{0}^{2}\left(\begin{array}{l}n \\ 2\end{array}\right) p(1-p) \leq 8 \mu_{0}^{2} n^{2} p=\sigma^{2}$. Let $a=\mu / 3$. Using that $k_{0}>1 / p$ and $\mu / \mu_{0}=(1+o(1)) n^{2} / k_{0}^{2}$, it is easy to check that $4 \mu_{0} a \leq 2 \sigma^{2}=16 \mu_{0}^{2} n^{2} p$. Note that by Corollary 2.3 , if $X \leq 3 \mu / 5$ then we also have that $X-\mathbb{E}[X]<-a$. Therefore, the desired estimate for the lower tail of $X$ follows from the above inequality of [1].

Remark. Using the same proof one can also obtain estimates on the upper tail of $X$ and in particular show that for any fixed $\delta>0$

$$
\operatorname{Pr}[|X-\mathbb{E}[X]|>\delta \mu]<2 e^{-\frac{\delta^{2} \mu^{2}}{40 \mu_{0}^{2} n^{2} p}} .
$$

Finally we are ready to prove the main result of this section, which roughly says that in the random graph $G_{n, p}$ independent sets of nearly maximal size are uniformly distributed in the following sense. Suppose we add to the random graph some set $E$ of $m$ edges. Consider a random subset of vertices of size $k_{0}$. By averaging, we expect that $2 m / n^{2}$ fraction of its pairs are edges from $E$. Our next lemma shows that with very high probability $G_{n, p}$ has an independent set of size $k_{0}$, which has this property up to a constant factor.

Lemma 2.4. If $n^{-1 / 3+\delta} \leq p \leq 1 / 2$ for some $\delta>0$, then with probability at least $1-e^{-n^{1+\delta}}$ the random graph $G_{n, p}$ has the following property. For every collection $E$ of $m$ edges, there is an independent set $I$ in $G_{n, p}$ of size $k_{0}$ such that I contains at most $7 k_{0}^{2} \frac{m}{n^{2}}$ edges of $E$. 
Proof. Let $\mathcal{I}$ be a largest collection of independent sets of size $k_{0}$ in random graph $G_{n, p}$ such that no pair of vertices belongs to more than $4 \mu_{0}$ of these sets. Recall that $\mu_{0} / \mu=(1+o(1)) k_{0}^{2} / n^{2}$. Also, by the definition of $k_{0}$, it is easy to check that $k_{0} \leq \frac{2}{p} \log n$. Together this implies that

$$
\frac{\mu^{2}}{300 \mu_{0}^{2} n^{2} p} \geq \frac{n^{2}}{400 k_{0}^{4} p} \geq \frac{n^{2} p^{3}}{10^{4} \log ^{4} n}>n^{1+\delta} .
$$

Therefore, by Lemma 2.1 we have that with probability at least $1-e^{-n^{1+\delta}}$ the size of $\mathcal{I}$ is at least $3 \mu / 5$. Consider an auxiliary bipartite graph $H$ with parts $\mathcal{I}$ and $E$ in which independent set $I \in \mathcal{I}$ is adjacent to edge $(u, v) \in E$ iff both vertices $u, v$ belong to $I$. By the definition of $\mathcal{I}$, every edge $(u, v) \in E$ is contained in at most $4 \mu_{0}$ sets from $\mathcal{I}$. Therefore the number of edges $e(H)$ is bounded by $4 \mu_{0} \mathrm{~m}$. Thus there is an independent set $I \in \mathcal{I}$, whose degree in $H$ is at most $e(H) /|\mathcal{I}|$. This $I$ contains at most

$$
\frac{e(H)}{|\mathcal{I}|} \leq \frac{4 \mu_{0} m}{|\mathcal{I}|} \leq \frac{20 \mu_{0} m}{3 \mu} \leq 7 k_{0}^{2} \frac{m}{n^{2}}
$$

edges from $E$.

Remark. As pointed out by an anonymous referee, one can give a slightly shorter proof of the last lemma relying more on the results of [11], but we prefer to include the proof above which is more transparent, even if a bit longer.

\section{Global and local resilience of the chromatic number}

In this section we prove our main results. We start by recalling several additional facts used in the proofs. The first is the following classical theorem of Turán (see e.g., [2] p. 95), which provides a lower bound for the size of a maximum independent set in a graph.

Lemma 3.1. Let $G$ be graph on $n$ vertices with $e(G)$ edges. Then the independence number $\alpha(G)$ of $G$ satisfies

$$
\alpha(G) \geq \frac{n^{2}}{2 e(G)+n} .
$$

We also need the following simple lemma which estimates the number of edges spanned by small subsets of a random graph. 
Lemma 3.2. Let $n^{-1 / 3} \leq p \leq 1 / 2$ and let $\epsilon$ be a positive constant. Then a.a.s. every subset of $G_{n, p}$ of size $s \leq \frac{\epsilon n}{16 \log (n p)}$ contains at most $\frac{\epsilon n p}{8 \log (n p)} s$ edges.

Proof. Define $r=\frac{\epsilon n}{16 \log (n p)}$. The probability of existence of a subset violating the assertion of the lemma is at most

$\sum_{s=r p}^{r}\left(\begin{array}{l}n \\ s\end{array}\right)\left(\begin{array}{c}\left(\begin{array}{c}s \\ 2\end{array}\right) \\ 2 s r p\end{array}\right) p^{2 s r p} \leq \sum_{s=r p}^{r}\left(\frac{e n}{s}\left(\frac{e s}{4 r p}\right)^{2 r p} p^{2 r p}\right)^{s} \leq \sum_{s=r p}^{r}\left(n\left(\frac{e}{4}\right)^{2 r p}\right)^{s}=o(1)$.

Here we used that $r p>\sqrt{n}$ together with the well known fact that $\left(\begin{array}{l}a \\ b\end{array}\right) \leq$ $(e a / b)^{b}$.

Finally, recall the simple fact that any graph with a chromatic number of at least $r$ must have a subgraph with minimum degree $r-1$.

Proof of Theorem 1.2. Let $E$ be an arbitrary set of $m=2^{-12} \epsilon^{2} n^{2} / \log _{b}^{2}(n p)$ edges, suppose it has been added to $G_{n, p}$, and put $G=G_{n, p} \cup E$. Let $b=1 /(1-p)$ and let $k=2 \log _{b}\left(n p / \log ^{3} n\right)=(1+o(1)) 2 \log _{b}(n p)$. Since every subset of $G_{n, p}$ is a random graph itself and the number of subsets is at most $2^{n}$, using Lemma 2.4 together with the union bound, we obtain that a.a.s. every subset of $G_{n, p}$ of size $s \geq n / \log ^{2} n$ has an independent set $I$ of size at least $k$ which contains at most $7 k_{0}^{2} \frac{m}{s^{2}} \leq 8 k^{2} \frac{m}{s^{2}}$ edges of $E$. Repeatedly apply the following procedure until the remaining graph has at most $\frac{\epsilon n}{16 \log (n p)}$ vertices. Given a current subset, which has $s$ vertices, find in it an independent set $I$ of $G_{n, p}$ of size at least $k$ which contains at most $8 k^{2} \frac{m}{s^{2}}$ edges of $E$. Apply Lemma 3.1 to the induced subgraph $G[I]$ to find an independent set of $G$ of size $\frac{k^{2}}{16 k^{2} m / s^{2}+k}$. Color it by a new color, remove its vertices from $G$ and continue.

If the current subgraph of $G$ has $s \geq 2^{-i} n$ vertices, then we can find in it an independent set of size at least $\frac{k^{2}}{2^{2 i+4} k^{2} m / n^{2}+k}$. Therefore if we start with at most $2^{-i+1} n$ vertices, then after pulling out

$$
\chi_{i} \leq 2^{-i} n / \frac{k^{2}}{2^{2 i+4} k^{2} m / n^{2}+k}=2^{i+4} \frac{m}{n}+2^{-i} \frac{n}{k}
$$

independent sets we will remain with less than $2^{-i} n$ vertices. Let $i_{0}$ be such that $2^{i_{0}}=16 \epsilon^{-1} \log (n p)$. Summing up for all $1 \leq i \leq i_{0}$ we obtain that we can color all but $\frac{\epsilon n}{16 \log (n p)}$ vertices of $G$ using only 


$$
\begin{aligned}
\sum_{i=1}^{i_{0}} \chi_{i} & =\sum_{i=1}^{i_{0}}\left(2^{i+4} \frac{m}{n}+2^{-i} \frac{n}{k}\right) \\
& \leq 2^{i_{0}+5} \frac{m}{n}+n / k \leq 2^{9} \epsilon^{-1} \log (n p) \frac{2^{-12} \epsilon^{2} n}{\log _{b}^{2}(n p)}+n / k \\
& \leq \frac{\epsilon}{4} \cdot \frac{\log (n p)}{\log _{b}(n p)} \cdot n / k+n / k \leq(1+\epsilon / 4) n / k
\end{aligned}
$$

colors. Next we prove that the remaining $\frac{\epsilon n}{16 \log (n p)}$ vertices of $G$ can be colored by at most $r=\frac{\epsilon n}{3 \log _{b}(n p)}$ additional colors.

Indeed suppose that the remaining vertices form a graph with a chromatic number more than $r$. Then this graph contains a subgraph $G^{\prime}$ with minimum degree at least $r-1$. Denote by $s$ the number of vertices of $G^{\prime}$ and note that $r \leq s \leq \frac{\epsilon n}{16 \log (n p)}$ vertices. The number of edges in $G^{\prime}$ is at least $s(r-1) / 2$. On the other hand, using that $\frac{1}{p} \log (n p) \geq \log _{b}(n p)$ together with Lemma 3.2, we conclude that the number of edges in $G^{\prime}$ is at most

$$
\begin{aligned}
\frac{\epsilon n p}{8 \log (n p)} s+|E| & \leq \frac{\epsilon n}{8 \log _{b}(n p)} s+\frac{2^{-12} \epsilon^{2} n^{2}}{\log _{b}^{2}(n p)} \leq \frac{\epsilon n}{8 \log _{b}(n p)} s+\frac{2^{-10} \epsilon n}{\log _{b}(n p)} s \\
& <s \frac{\epsilon n}{7 \log _{b}(n p)}<\frac{s(r-1)}{2} .
\end{aligned}
$$

This contradiction shows that any $\frac{\epsilon n}{16 \log (n p)}$ vertices of $G$ can be colored by at most $\frac{\epsilon n}{3 \log _{b}(n p)}$ colors. Therefore the chromatic number of $G$ is at most $(1+\epsilon / 4) n / k+\epsilon n /\left(3 \log _{b} n\right)<(1+\epsilon) \frac{n}{2 \log _{b}(n p)}$, completing the proof.

Proof of Theorem 1.3. The proof of this result follows along the same lines as the one for global resilience. Let $H$ be a graph with maximum degree $\Delta \leq 2^{-8} \epsilon_{\frac{n}{\log _{b}(n p) \log \log n}}$ and let $G=G_{n, p} \cup H$. Set $b=1 /(1-p)$ and $k=2 \log _{b}\left(n p / \log ^{3} n\right)=(1+o(1)) 2 \log _{b}(n p)$. Let $S$ be a subset of $G_{n, p}$ of size $s=|S| \geq n / \log ^{2} n$ and let $e(H[S])$ be the number of edges of $H$ spanned by $S$. Since $H$ has bounded maximum degree we have that $e(H[S]) \leq \Delta s / 2$. We can again assume, by Lemma 2.4, that every such subset $S$ has an independent set $I$ of $G_{n, p}$ of size at least $k$ which contains at most

$$
7 k_{0}^{2} \frac{e(H[S])}{s^{2}} \leq 8 k^{2} \frac{e(H[S])}{s^{2}} \leq \frac{4 k^{2} \Delta}{s}
$$

edges of $H$. Applying Lemma 3.1 to the induced subgraph $G[I]$, we find in it an independent set of $G$ of size $\frac{k^{2}}{8 k^{2} \Delta / s+k}$. Repeatedly color every such 
independent set by a new color and remove it from $G$ until the remaining graph has at most $\frac{n}{\log ^{2} n}$ vertices.

If the current subgraph of $G$ has $s \geq 2^{-i} n$ vertices, then we can find in it an independent set of size at least $\frac{k^{2}}{2^{i+3} k^{2} \Delta / n+k}$. Therefore if we start with at most $2^{-i+1} n$ vertices, then after pulling out

$$
\chi_{i} \leq 2^{-i} n / \frac{k^{2}}{2^{i+3} k^{2} \Delta / n+k}=8 \Delta+2^{-i} \frac{n}{k}
$$

independent sets, we will remain with less than $2^{-i} n$ vertices. Let $i_{0}$ be such that $2^{i_{0}}=\log ^{2} n$, then $i_{0} \leq 3 \log \log n$. Summing up for all $1 \leq i \leq i_{0}$ we obtain that we can color all but $\frac{n}{\log ^{2} n}$ vertices of $G$ using only

$$
\begin{aligned}
\sum_{i=1}^{i_{0}} \chi_{i} & =\sum_{i=1}^{i_{0}}\left(8 \Delta+2^{-i} \frac{n}{k}\right) \leq \frac{n}{k}+8 \Delta i_{0} \leq \frac{n}{k}+24 \log \log n \frac{2^{-8} \epsilon n}{\log _{b}(n p) \log \log n} \\
& \leq(1+\epsilon / 4) \frac{n}{k}
\end{aligned}
$$

colors.

Consider any subset $S$ of $s \leq \frac{n}{\log ^{2} n}$ vertices of $G$. By Lemma 3.2 it has at most $\frac{\epsilon n p}{8 \log (n p)} s$ edges of $G_{n, p}$. The number of edges of $H$ inside $S$ is clearly at most $\Delta s / 2$. Therefore there are at most $\left(\frac{\epsilon n p}{8 \log (n p)}+\Delta / 2\right) s$ edges in the subgraph of $G$ induced by $S$. Using that $\frac{1}{p} \log (n p) \geq \log _{b}(n p)$ we conclude that $G[S]$ has a vertex of degree at most

$$
2 e(G[S]) / s \leq \frac{\epsilon n p}{4 \log (n p)}+\Delta<\frac{\epsilon n}{2 \log _{b}(n p)}+\frac{2^{-8} \epsilon n}{\log _{b}(n p) \log \log n}<\frac{3 \epsilon n}{5 k}-1 .
$$

This shows that we can color the remaining $\frac{n}{\log ^{2} n}$ vertices in $3 \epsilon n /(5 k)$ colors and the whole graph $G$ by $(1+\epsilon / 4+3 \epsilon / 5) \frac{n}{k}<(1+\epsilon) \frac{n}{2 \log _{b}(n p)}$ colors.

\section{Concluding remarks and open problems}

We have studied the global and local resilience of random graphs with respect to the property of having a chromatic number close to its typical value. Our bounds for global resilience are tight up to a constant factor, but the ones for the local case are only tight up to a $\log \log n$ factor. It seems plausible to conjecture that the assertion of Theorem 1.3 holds even when the $\log \log n$ term is omitted in the hypothesis. It is also possible that Theorem 1.2 can be 
strengthened, and that the most economical way to increase the chromatic number of the random graph $G_{n, p}$ by a factor of $(1+\epsilon)$ is to construct an appropriately large clique in it. If this is the case, then one has to add to $G_{n, p}$, a.a.s., more than $n^{2} /\left(16 \log _{b}^{2} n\right)$ edges in order to increase its chromatic number by a factor of $(1+\epsilon)$, for any fixed $\epsilon>0$ and sufficiently large $n$. This remains open. It may also be interesting to estimate the minimum number of edges that have to be added to $G_{n, p}$ in order to increase the chromatic number by a lower order term. This is related to the question of estimating the concentration of the chromatic number of random graphs, and appears to be difficult.

\section{References}

[1] N. Alon, J. H. Kim and J. Spencer, Nearly perfect matchings in regular simple hypergraphs, Israel J. Math. 100 (1997), 171-187. MR1469109

[2] N. Alon and J. Spencer, The Probabilistic Method, 3rd edition, Wiley, New York, 2008. MR2437651

[3] J. Balogh, B. Csaba and W. Samotij, Local resilience of almost spanning trees in random graphs, Random Structures and Algorithms, to appear.

[4] B. Bollobás, The chromatic number of random graphs, Combinatorica 8 (1988), 49-55. MR0951992

[5] B. Bollobás, Random Graphs, 2nd edition, Cambridge Studies in Advanced Mathematics 73, Cambridge University Press, Cambridge, 2001. MR1864966

[6] J. Böttcher, Y. Kohayakawa and A. Taraz, Almost spanning subgraphs of random graphs after adversarial edge removal, Electronic Notes in Discrete Mathematics 35 (2009), 335-340. MR2579452

[7] D. Dellamonica, Y. Kohayakawa, M. Marciniszyn and A. Steger, On the resilience of long cycles in random graphs, Electron. J. Combin. 15 (2008), R32. MR2383452

[8] A. Frieze and M. Krivelevich, On two Hamilton cycle problems in random graphs, Israel J. Math. 166 (2008), 221-234. MR2430433

[9] S. Janson, T. Łuczak and A. Ruciński, Random Graphs, John Wiley and Sons, New York, 2000. MR1782847

[10] J.H. Kim, B. Sudakov and V. Vu, On the asymmetry of random regular graphs and random graphs, Random Structures Algorithms 21 (2002), 216-224. MR1945368 
[11] M. Krivelevich, B. Sudakov, V. Vu and N. Wormald, On the probability of independent sets in random graphs, Random Structures and Algorithms 22 (2003), 1-14. MR1943855

[12] M. Krivelevich, C. Lee and B. Sudakov, Resilient pancyclicity of random and pseudo-random graphs, SIAM J. of Discrete Math. 24 (2010), 1-16. MR2600649

[13] L. Lovász, Combinatorial Problems and Exercises, 2nd edition, AMS Chelsea Publishing, 2007. MR2321240

[14] T. Łuczak, The chromatic number of random graphs, Combinatorica 11 (1991), 45-54. MR1112273

[15] B. Sudakov and V. Vu, Local resilience of graphs, Random Structures and Algorithms 33 (2008), 409-433. MR2462249

Noga AlON

Schools of Mathematics and Computer Science

SACKLER FACUlty of EXACT ScIEnCES

Tel Aviv University

Tel Aviv 69978, IsRAel

E-mail address: nogaa@tau.ac.il

BENNY SUDAKOV

Department of Mathematics

UCLA, LOS ANGELES

CA 90095, USA

E-mail address: bsudakov@math.ucla.edu

Received February 8, 2010 